Sharif University of Technology
Scientia Iranica
Transactions E: Industrial Engineering
hCIENTIA

\title{
Single-valued neutrosophic linguistic reducible weighted MSM for tourism mobile e-commerce service quality evaluation
}

\author{
X. Peng* \\ School of Information Science and Engineering, Shaoguan University, Shaoguan, 512005, China. \\ Received 20 December 2018; received in revised form 24 February 2020; accepted 8 June 2020
}

\author{
KEYWORDS \\ Single-valued \\ neutrosophic linguistic \\ set; \\ Reducibility; \\ Idempotency; \\ SVNLRWMSM; \\ SVNLRWDMSM.
}

\begin{abstract}
The Tourism Mobile E-commerce Service Quality Evaluation (TMESQE) is of great concern to enterprises for enriching the service content of the enterprise and improving its market competitiveness. The key issue is the tremendous vagueness and reciprocity for TMESQE. The Maclaurin Symmetric Mean (MSM), as a vital fusion approach, can capture the reciprocity between multiple given arguments more effectually. Amount of Weighted MSM (WMSM) has been presented for dealing with various neutrosophic information integration issues because the arguments are hourly interoperable. However, these kinds of WMSM operators are out of the reducibility or idempotency. To handle two problems above, we introduce Single-Valued Neutrosophic Linguistic Reducible Weighted MSM (SVNLRWMSM) operator and Single-Valued Neutrosophic Linguistic Reducible Weighted Dual MSM (SVNLRWDMSM) operator. In the meantime, the diverting properties and certain peculiar cases of developed operators are discussed. Therefore, we explore two Multi-Criteria Decision-Making (MCDM) algorithm based on SVNLRWMSM and SVNLRWDMSM for dealing with the TMESQE issue, along with the sensitivity analysis of various values on final ordering. Conclusively, the comparison of some existing algorithms has been conducted for showing their availability.
\end{abstract}

(C) 2022 Sharif University of Technology. All rights reserved.

\section{Introduction}

The rapid development of mobile communication technology and Internet Technology provides a favorable environment for mobile e-commerce applications in people's lives and in various industries. In recent years, the number of mobile users, including smartphones, has explosively increased by the 42 nd China Internet

\footnotetext{
*. Tel.: 86-15889857163

E-mail address: 952518336@qq.com,

pengxindong@sgu.edu.cn (X.Peng)
}

doi: $10.24200 /$ sci. 2020.52511 .2749 network development statistics report [1]. The number of Internet users in China is 802 million till June 2018, among which the number of mobile Internet users is 788 million, accounting for $98.3 \%$ of the total number of Internet users. It can be seen that people's life is more and more inclined to using mobile. Moreover, under the background of rapid economic development and continuous improvement of residents' consumption level in China, tourism has gradually become a popular leisure and entertainment mode, and the scale of tourism consumption in China is increasing year by year. The China tourism academy released the tourism economic data report for the first half of 2018, which showed that the number of domestic tourists reached 2.826 
billion, up by $11.4 \%$ from the same period last year [2]. Tourism consumers are also increasingly diversified and self-service in their demands for tourism. They begin to choose tourism products according to their own preferences, time, cost, and other factors. In particular, in the context of mobile e-commerce, information access is more convenient and the individualization of tourism groups becomes more prominent. Under these two backgrounds, mobile e-commerce is widely used in combination with the tourism industry due to its unique advantages such as timeliness, convenience, and personalization. The all-round development of Tourism Mobile E-commerce (TME) has become an inevitable trend. The use of TME runs through the whole process of tourism. Users can complete travel route planning, accommodation, and catering reservations before tourism, obtain real-time tourism information, and formulate or change tourism modes and tourism and end of tourism at any time to ensure tourism. The mobile e-commerce platform can control the itinerary by itself, and people begin to enjoy the convenience and fun provided by the TME.

Due to the close cooperation between mobile ecommerce and tourism in recent years, the tourism market in these years is dominated by the expansion of TME. More and more companies join in this big market. In the fierce competition environment, service quality problems begin to appear frequently. According to the complaint platform of people's tourism website 3.15 , there are many complaints from tourism consumers about the service of the TME platform. For example, there is a big gap between the description on the Internet and the tourism products provided; there is a problem with the ticket refund and alteration on the website; and the service commitment of fraudulent marketing activities cannot be fulfilled. The emergence of these problems has a negative impact on users' experience and feelings, reduces users' satisfaction, and puts tourism service enterprises at a disadvantage. Compared to the conventional e-commerce service, the mobile e-commerce service of tourism has more unstable factors and higher requirements on service quality $[3,4]$. Therefore, the construction of the evaluation model based on user experience is critical to improving both the user experience and the service quality of the company. Nevertheless, the increasingly intricate decision-making environments and indecisive Decision Maker (DM) have trouble in presenting preference information with uncertain information when solving the above Multi-Criteria Decision-Making (MCDM) issues.

Single-Valued Neutrosophic Linguistic Set (SVNLS), initiatively conceived by Ye [5], is perceived as a more underlying measure for depicting the indeterminacy of quantitative and qualitative evaluation information, which is a generalization of materialization Neutrosophic Set (NS) [6] (named Single- Valued Neutrosophic Set (SVNS) [7]). It constitutes a linguistic portion (qualitative) and a fuzzy portion (quantitative) where the fuzzy portion is employed in describing the confidence degree of linguistic value in the linguistic portion. SVNLS is an effective instrument for presenting preference information in the Tourism Mobile E-commerce Service Quality Evaluation (TMESQE). For instance, when a DM or an expert is required to evaluate a TME company, she or he offers a qualitative assessment (e.g., the TME product is excellent) in place of a numerical scale. Moreover, the degree of which she or he considers as the evaluation is true ranges between 0 and 1 such as 0.6. The degree of which she or he considers as the evaluation is false ranges between 0 and 1 , such as 0.3 . The degree of which she or he does not ascertain about the evaluation is a decimal between 0 and 1 , such as 0.4 . In addition, these three degrees are independent of each other, without limitation in the sum of three degrees. SVNSs can describe information with respect to the reliance of the evaluation faultlessly and SVNLSs can present the evaluations in TME company selection triumphantly. Up to now, SVNLS has attracted much attention and obtained certain exciting achievements [8-11]. Tian et al. [12] developed some power aggregation for SVNLS in the green product design selection process. Ye [13] presented the SVNL weighted arithmetic average and SVNL weighted geometric average for dealing with the selection of flexible manufacturing system. Tian et al. [14,15] proposed the Single-Valued Neutrosophic Linguistic Normalized Weighted Bonferroni Mean (SVNLNWBM) operator for dealing with the issue of investment appraisal project selection, which can seize the relevancy of given arguments. Tan et al. [16] presented the generalized form of the SVNL aggregation operators with greater flexibility in employing the complex real problems. Wang et al. [17] combined Single-valued Neutrosophic Cloud (SNC) notion with the neutrosophic linguistic Maclaurin Symmetric Mean (MSM) operator [18] for solving the hotel selection.

However, it just captures the interaction among a constant number of parameters using the above Aggregation Operators (AOs) [12-18]. For instance, the Heronian Mean (HM) operator only captures the correlation between two parameters. For boosting the elasticity of information integration, Maclaurin [19] presented the MSM operator that can seize the interaction among any number of parameters. Qin and Liu [20] initially combined MSM operator with intuitionistic fuzzy environment in an uncertain field and initiated the weighted intuitionistic fuzzy MSM for aggregating decision evaluation information. At present, there are many generalizations of MSM operators by employing them in different indeterminate environments [21- 
33]. According to the research of Weighted MSM (WMSM) operators in various uncertain environments, two counter-intuitive issues can be easily seen in the following: (1) When weight information in entire parameters is the same, the corresponding WMSM operators [21-33] fail to degenerate into consistent MSM operators, representing a key peculiarity of the traditional weighted operators; and (2) Such kinds of WMSM operators [21-31] are out of idempotency. That is to say, it is illogical that the weighted average value of certain selfsame aggregated parameters relies on corresponding weight values. Illuminated by Reducible WMSM (RWMSM) operator and Reducible Weighted Dual MSM (RWDMSM) operator [34], we combine the above-mentioned operators with SVNLS for fusing single-valued neutrosophic linguistic information and dealing with the corresponding MCDM issues by considering the virtues of both.

The above discussion comes down to the main four targets in the following:

1. Introduce two new kinds of AOs (Single-Valued Neutrosophic Linguistic Reducible Weighted MSM (SVNLRWMSM) and Single-Valued Neutrosophic Linguistic Reducible Weighted Dual MSM (SVNLRWDMSM)) for integrating the decision preferences of DMs or experts;

2. Present two MCDM algorithm based on SVNLRWMSM and SVNLRWDMSM for dealing with the TME service quality issue;

3. Discuss the sensitivity analysis of different parameter values on the conclusive ranking;

4. Construct a comprehensive appraisal system for TME service quality.

The remaining part of the paper is listed in the following. In Section 2, we simply present fundamental notions of SVNLS, MSM, RWMSM, Dual Maclaurin Symmetric Mean (DMSM), and RWDMSM operators. In Section 3, the SVNLRWMSM and SVNLRWDMSM operators are presented with their interesting properties. In Section 4, two MADM algorithms based on SVNLRWMSM operator and SVNLRWDMSM operator are given with a Single- Valued Neutrosophic Linguistic Number (SVNLN). In addition, we employ an example of TME service quality to state the feasibility of MCDM approaches by presenting the effect of diverse argument values on final ordering of the alternatives. In Section 5, a comparison with certain existing MCDM algorithms is presented. Also, the characteristic comparisons of diverse SVNL AOs and various inconclusive environment are made. Finally, Section 6 offers the conclusive remarks.

\section{Preliminaries}

\subsection{Single-Valued Neutrosophic Linguistic Set $(S V N L S)$}

Definition 1 [5]. Let $X$ be the universe of discourse, with a crowd of elements in $X$ signified by $x$. Let $S=$ $\left\{s_{0}, s_{1}, \ldots, s_{l}\right\}$ be a limited and total ordered discrete Linguistic Term Set (LTS). The SVNLS can be denoted in the following:

$$
A=\left\{<s_{\theta(x)},\left(T_{A}(x), I_{A}(x), F_{A}(x)\right)>\mid x \in X\right\},
$$

where $x \in X, s_{\theta(x)} \in S, T_{A}(x) \in[0,1], I_{A}(x) \in[0,1]$, and $F_{A}(x) \in[0,1]$. The $T_{A}(x), I_{A}(x)$ and $F_{A}(x)$ are the truth-membership, indeterminate-membership, and false-membership of $x$ in $X$ to $s_{\theta(x)}$, respectively. In addition, $0 \leq T_{A}(x)+I_{A}(x)+F_{A}(x) \leq 1$ exists for any $x \in X$. Moreover, $a=<s_{\theta(x)},\left(T_{A}(x), I_{A}(x), F_{A}(x)\right)>$ is called Single-Valued Neutrosophic Linguistic Number (SVNLN). For simplicity, an SVNLN is defined as $a=<s_{\theta(a)},\left(T_{a}, I_{a}, F_{a}\right)>$.

Definition 2 [5]. Let $x=<s_{\theta(x)},\left(T_{x}, I_{x}, F_{x}\right)>$ and $y=<s_{\theta(y)},\left(T_{y}, I_{y}, F_{y}\right)>$ be two SVNLNs and $\lambda>0$; then, operations are shown in the following:

1. $x^{c}=<s_{l-\theta(x)},\left(F_{x}, 1-I_{x}, T_{x}\right)>$;

2. $x \oplus y=<s_{\theta(x)+\theta(y)},\left(T_{x}+T_{y}-T_{x} * T_{y}, I_{x} * I_{y}, F_{x} *\right.$ $\left.F_{y}\right)>$;

3. $x \otimes y=<s_{\theta(x) \theta(y)},\left(T_{x} * T_{y}, I_{x}+I_{y}-I_{x} * I_{y}, F_{x}+\right.$ $\left.F_{y}-F_{x} * F_{y}\right)>$;

4. $\lambda x=<s_{\lambda \theta(x)},\left(1-\left(1-T_{x}\right)^{\lambda},\left(I_{x}\right)^{\lambda},\left(F_{x}\right)^{\lambda}\right)>$;

5. $x^{\lambda}=<s_{\theta^{\lambda}(x)},\left(\left(T_{x}\right)^{\lambda}, 1-\left(1-I_{x}\right)^{\lambda}, 1-\left(1-F_{x}\right)^{\lambda}\right)>$.

Definition 3 [12]. Let $x=<s_{\theta(x)},\left(T_{x}, I_{x}, F_{x}\right)>$ be an SVNLN. Then, the score function $s(x)$, the accuracy function $a(x)$, and the certainty function $c(x)$ are suggested in the following:

1. $s(x)=\theta(x)\left(T_{x}+2-I_{x}-F_{x}\right)$;

2. $a(x)=\theta(x)\left(T_{x}-F_{x}\right)$;

3. $c(x)=\theta(x) T_{x}$.

For any two SVNLNs $x, y$ :

1. When $s(x)>s(y)$, then $x \succ y$;

2. When $s(x)=s(y)$ and $a(x)>a(y)$, then $x \succ y$;

3. When $s(x)=s(y)$ and $a(x)=a(y)$;

If $c(x)>c(y)$, then $x \succ y$;

If $c(x)=c(y)$, then $x \sim y$.

\subsection{Reducible Weighted Maclaurin Symmetric Means ( $R W M S M$ )}

The MSM, developed by Maclaurin [19], can acquire the reciprocity among multiple given arguments more efficaciously. For now, the MSM is used for integrating vague evaluation information. 
Definition 4 [19]. Let $x_{i}(i=1,2, \cdots, n)$ be a set of nonnegative real numbers, and $k=1,2, \cdots, n$. Then, MSM operator is signified as follows:

$$
\begin{aligned}
& \operatorname{MSM}^{(k)}\left(x_{1}, x_{2}, \cdots, x_{n}\right)= \\
& \left(\frac{\sum_{1 \leq i_{1}<\cdots<i_{k} \leq n} \prod_{j=1}^{k} x_{i_{j}}}{C_{n}^{k}}\right)^{1 / k},
\end{aligned}
$$

where $\left(i_{1}, i_{2}, \cdots, i_{k}\right)$ is ergodic for all the $k$ permutations of $(1,2, \cdots, n)$ and $C_{n}^{k}$ is the binomial coefficient meeting $C_{n}^{k}=\frac{n !}{k !(n-k) !}$.

Definition 5 [21]. Let $x_{i}(i=1,2, \cdots, n)$ be a set of nonnegative real numbers and $k=1,2, \cdots, n$; then, DMSM operator is suggested as follows:

$$
\begin{aligned}
& \operatorname{DMSM}^{(k)}\left(x_{1}, x_{2}, \cdots, x_{n}\right)= \\
& \left(\frac{\sum_{1 \leq i_{1}<\cdots<i_{k} \leq n} \prod_{j=1}^{k} x_{i_{j}}}{C_{n}^{k}}\right)^{1 / k},
\end{aligned}
$$

where $\left(i_{1}, i_{2}, \cdots, i_{k}\right)$ is ergodic for all the $k$ permutations of $(1,2, \cdots, n)$ and $C_{n}^{k}$ is the binomial coefficient meeting $C_{n}^{k}=\frac{n !}{k !(n-k) !}$.

In the sake of addressing the problems of idempotency and reducibility, Shi and Xiao [34] introduced RWDMSM and RWMSM.

Definition 6 [34]. Let $x_{i}(i=1,2, \cdots, n)$ be a series of nonnegative real numbers, $k=1,2, \cdots, n$, and $W=\left(w_{1}, w_{2}, \cdots, w_{n}\right)^{T}$ with $w_{i} \in[0,1]$ and $\sum_{i=1}^{n} w_{i}=$ 1. Then, the RWMSM operator is suggested in the following:

$$
\begin{aligned}
& \operatorname{RWMSM}^{(k)}\left(x_{1}, x_{2}, \cdots, x_{n}\right)= \\
& \left(\frac{\sum_{1 \leq i_{1}<\cdots<i_{k} \leq n}\left(\prod_{j=1}^{k} w_{i_{j}}\right)\left(\prod_{j=1}^{k} x_{i_{j}}\right)}{\sum_{1 \leq i_{1}<\cdots<i_{k} \leq n} \prod_{j=1}^{k} w_{i_{j}}}\right)^{1 / k} .
\end{aligned}
$$

Definition 7 [34]. Let $x_{i}(i=1,2, \cdots, n)$ be a series of nonnegative real numbers, $k=1,2, \cdots, n$, and $W=$ $\left(w_{1}, w_{2}, \cdots, w_{n}\right)^{T}$ with $w_{i} \in[0,1]$ and $\sum_{i=1}^{n} w_{i}=1$; then, RWDMSM operator is given as follows:

$$
\begin{aligned}
& \operatorname{RWDMSM}^{(k)}\left(x_{1}, x_{2}, \cdots, x_{n}\right)= \\
& \frac{\prod_{1 \leq i_{1}<\cdots<i_{k} \leq n}\left(\sum_{j=1}^{k} x_{i_{j}}\right) \frac{\sum_{j=1}^{\sum_{1 \leq i_{1}<\cdots<i_{k} \leq n} w_{i_{j}} \sum_{j=1}^{k} w_{i_{j}}}}{k}}{k} .
\end{aligned}
$$

\section{Single-valued Neutrosophic Linguistic Reducible Weighted MSMs (SVNLRWMSMs)}

\subsection{SVNLRWMSM operator}

Definition 8. Let $x_{i}=<s_{\theta\left(x_{i}\right)},\left(T_{x_{i}}, I_{x_{i}}, F_{x_{i}}\right)>$ $(i=1,2, \cdots, n)$ be a set of SVNLNs and let $W=$ $\left(w_{1}, w_{2}, \cdots, w_{n}\right)^{T}$ be a weight vector where $w_{i} \in[0,1]$ and $\sum_{i=1}^{n} w_{i}=1$. The SVNLRWMSM, $\Omega^{n} \rightarrow \Omega$ : an SVNLRWMSM operator is given as follows:

$$
\begin{aligned}
& \operatorname{SVNLRWMSM}^{(k)}\left(x_{1}, x_{2}, \cdots, x_{n}\right)= \\
& \left(\frac{\sum_{1 \leq i_{1}<\cdots<i_{k} \leq n}\left(\prod_{j=1}^{k} w_{i_{j}}\right)\left(\prod_{j=1}^{k} x_{i_{j}}\right)}{\sum_{1 \leq i_{1}<\cdots<i_{k} \leq n} \prod_{j=1}^{k} w_{i_{j}}}\right)^{1 / k},
\end{aligned}
$$

where $\Omega$ is the set of entire SVNLNs and then, SVNLRWMSM is named the Single-Valued Neutrosophic Linguistic RWMSM operator.

Based on the operational laws of SVNLNs defined in Definition 2 and Eq. (6), we can obtain the aggregated result presented in Theorem 1.

Theorem 1. Let $x_{i}=<s_{\theta\left(x_{i}\right)},\left(T_{x_{i}}, I_{x_{i}}, F_{x_{i}}\right)>$ $(i=1,2, \cdots, n)$ be a series of $S V N L N s$ and let $W=\left(w_{1}, w_{2}, \cdots, w_{n}\right)^{T}$ be a weight vector where $w_{i} \in[0,1]$ and $\sum_{i=1}^{n} w_{i}=1$; then, the final result of SVNLRWMSM operator is still an SVNLN.

Eq. (7) is shown in Box I.

Proof. According to Definition 2, we can have:

$$
\begin{aligned}
\prod_{j=1}^{k} x_{i_{j}}= & \left\langle s \prod_{j=1}^{k} \theta\left(x_{i_{j}}\right)\right. \\
& \left.1-\left(\prod_{j=1}^{k}\left(1-I_{x_{i_{j}}}\right), 1-\prod_{j=1}^{k}\left(1-F_{x_{x_{i}}}\right)\right)\right\rangle,
\end{aligned}
$$

and:

$$
\begin{gathered}
\left(\prod_{j=1}^{k} w_{i_{j}}\right)\left(\prod_{j=1}^{k} x_{i_{j}}\right)=\left\langle s\left(\prod_{j=1}^{k} w_{i_{j}}\right)\left(\prod_{j=1}^{k} \theta\left(x_{i_{j}}\right)\right),\right. \\
\left(1-\left(1-\prod_{j=1}^{k} T_{x_{i_{j}}}\right)^{\prod_{j=1}^{k} w_{i_{j}}},\right. \\
\left(1-\prod_{j=1}^{k}\left(1-I_{x_{i_{j}}}\right)\right)^{\prod_{j=1}^{k} w_{i_{j}}}
\end{gathered}
$$




$$
\begin{aligned}
& \operatorname{SVNLRWMSM}^{(k)}\left(x_{1}, x_{2}, \cdots, x_{n}\right)=
\end{aligned}
$$

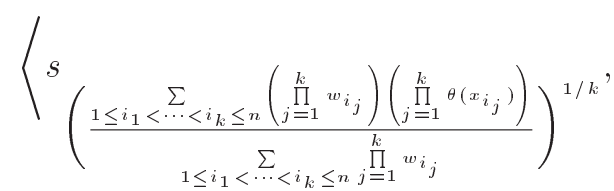

$$
\begin{aligned}
& \left(\left(1-\left(\prod_{1 \leq i_{1}<\cdots<i_{k} \leq n}\left(1-\prod_{j=1}^{k} T_{x_{i_{j}}}\right)^{\prod_{j=1}^{k} w_{i_{j}}}\right)^{\frac{1}{1 \leq i_{1}<\cdots<i_{k} \leq n j=1}{ }^{\sum_{j=1}^{k} w_{i_{j}}}}\right)^{1 / k},\right. \\
& 1-\left(1-\left(\prod_{1 \leq i_{1}<\cdots<i_{k} \leq n}\left(1-\prod_{j=1}^{k}\left(1-I_{x_{i_{j}}}\right)\right)^{\prod_{j=1}^{k} w_{i_{j}}}\right)^{\frac{1}{1 \leq i_{1}<\cdots<i_{k} \leq n j \prod_{j} \prod_{i_{j}}}}\right)^{1 / k}, \\
& \left.\left.1-\left(1-\left(\prod_{1 \leq i_{1}<\cdots<i_{k} \leq n}\left(1-\prod_{j=1}^{k}\left(1-F_{x_{i_{j}}}\right)\right)^{\prod_{j=1}^{k} w_{i_{j}}}\right)^{\frac{1}{1 \leq i_{1}<\cdots<i_{k} \leq n j=1} \prod_{j=1}^{k} w_{i_{j}}}\right)^{1 / k}\right)\right\rangle .
\end{aligned}
$$

Box I

$$
\left.\left.\left(1-\prod_{j=1}^{k}\left(1-F_{x_{i_{j}}}\right)\right)^{\prod_{j=1}^{k} w_{i_{j}}}\right)\right\rangle .
$$

Further:

$$
\begin{aligned}
& \sum_{1 \leq i_{1}<\cdots<i_{k} \leq n}\left(\prod_{j=1}^{k} w_{i_{j}}\right)\left(\prod_{j=1}^{k} x_{i_{j}}\right) \\
& =\left\langle s \sum_{1 \leq i_{1}<\cdots<i_{k} \leq n}\left(\prod_{j=1}^{k} w_{i_{j}}\right)\left(\prod_{j=1}^{k} \theta\left(x_{i_{j}}\right)\right),\right. \\
& \left(1-\prod_{1 \leq i_{1}<\cdots<i_{k} \leq n}\left(1-\prod_{j=1}^{k} T_{x_{i_{j}}}\right)^{\prod_{j=1}^{k} w_{i_{j}}},\right. \\
& \prod_{1 \leq i_{1}<\cdots<i_{k} \leq n}\left(1-\prod_{j=1}^{k}\left(1-I_{x_{i_{j}}}\right)\right)^{\prod_{j=1}^{k} w_{i_{j}}}, \\
& \left.\left.\prod_{1 \leq i_{1}<\cdots<i_{k} \leq n}\left(1-\prod_{j=1}^{k}\left(1-F_{x_{i_{j}}}\right)\right)^{\prod_{j=1}^{k} w_{i_{j}}}\right)\right\rangle .
\end{aligned}
$$

Consequently, the equation shown in Box II is obtained. In the end, we can obtain the equation shown in Box III, then we can know that Eq. (7) is right. Here the equation shown in Box IV can be easily obtained.

Consequently, we can consider that the aggregated result from Eq. (7) is an SVNLN.

Theorem 2 (Monotonicity). Let $x_{i}(i=1,2, \cdots, n)$ and $x_{i}^{\prime}(i=1,2, \cdots, n)$ be two sets of SVNLNs; if $x_{i}^{\prime}=<$ $s_{\theta\left(x_{i}^{\prime}\right)},\left(T_{x_{i}^{\prime}}, I_{x_{i}^{\prime}}, F_{x_{i}^{\prime}}\right)>, x_{i}=<s_{\theta\left(x_{i}\right)},\left(T_{x_{i}}, I_{x_{i}}, F_{x_{i}}\right)>$, $\theta\left(x_{i}\right) \geq \theta\left(x_{i}^{\prime}\right), T_{x_{i}} \geq T_{x_{i}^{\prime}}, I_{x_{i}} \leq I_{x_{i}^{\prime}}, F_{x_{i}} \leq F_{x_{i}^{\prime}}$ for $\forall i=$ $1,2, \cdots, n$, then:

$$
\begin{aligned}
& \operatorname{SVNLRWMSM}^{(k)}\left(x_{1}, x_{2}, \cdots, x_{n}\right) \\
& \quad \geq \operatorname{SVNLRWMSM}^{(k)}\left(x_{1}^{\prime}, x_{2}^{\prime}, \cdots, x_{n}^{\prime}\right) .
\end{aligned}
$$

Proof. Suppose that:

$\operatorname{SVNLRWMSM}^{(k)}\left(x_{1}, x_{2}, \cdots, x_{n}\right)=<s_{\theta},(T, I, F)>$,

$\operatorname{SVNLRWMSM}^{(k)}\left(x_{1}^{\prime}, x_{2}^{\prime}, \cdots, x_{n}^{\prime}\right)=<s_{\theta^{\prime}},\left(T^{\prime}, I^{\prime}, F^{\prime}\right)>$.

Since $\theta\left(x_{i}\right) \geq \theta\left(x_{i}^{\prime}\right)$, we can have $\prod_{j=1}^{k} \theta\left(x_{i_{j}}\right) \geq$ 


$$
\begin{aligned}
& \frac{\sum_{1 \leq i_{1}<\cdots<i_{k} \leq n}\left(\prod_{j=1}^{k} w_{i_{j}}\right)\left(\prod_{j=1}^{k} x_{i_{j}}\right)}{\sum_{1 \leq i_{1}<\cdots<i_{k} \leq n} \prod_{j=1}^{k} w_{i_{j}}}=\left\langle s \sum_{\substack{\left.1 \leq i_{1}<\cdots<i_{k} \leq n \\
\sum_{j=1}^{k} w_{i_{j}}\right)\left(\prod_{j=1}^{k} \theta\left(x_{i_{j}}\right)\right)}}^{\sum_{1 \leq i_{1}<\cdots<i_{k} \leq n} \prod_{j=1}^{k} w_{i_{j}}}\right. \\
& \left(1-\left(\prod_{1 \leq i_{1}<\cdots<i_{k} \leq n}\left(1-\prod_{j=1}^{k} T_{x_{i_{j}}}\right)^{\prod_{j=1}^{k} w_{i_{j}}}\right)^{\frac{1}{1 \leq i_{1}<\cdots<i_{k} \leq n} \prod_{j=1}^{\prod_{1}^{k} w_{i_{j}}}}\right. \\
& \left(\prod_{1 \leq i_{1}<\cdots<i_{k} \leq n}\left(1-\prod_{j=1}^{k}\left(1-I_{x_{i_{j}}}\right)\right)^{\prod_{j=1}^{k} w_{i_{j}}}\right)^{\frac{1}{1 \leq i_{1}<\cdots<i_{k} \leq n} \prod_{j=1}^{\prod_{1}^{k} w_{i_{j}}}} \\
& \left.\left.\left(\prod_{1 \leq i_{1}<\cdots<i_{k} \leq n}\left(1-\prod_{j=1}^{k}\left(1-F_{x_{i_{j}}}\right)\right)^{\prod_{j=1}^{k} w_{i_{j}}}\right)^{\frac{1}{1 \leq i_{1}<\cdots<i_{k} \leq n} \prod_{j=1}^{k} w_{i_{j}}}\right)\right\rangle .
\end{aligned}
$$

Box II

$\prod_{j=1}^{k} \theta\left(x_{i_{j}}^{\prime}\right)$. Furthermore, we have:

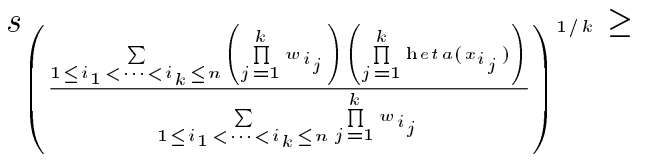

$$
\begin{aligned}
& s\left(\frac{\sum_{1 \leq i_{1}<\cdots<i_{k} \leq n}\left(\prod_{j=1}^{k} w_{i_{j}}\right)\left(\prod_{j=1}^{k} \theta\left(x_{i_{j}}^{\prime}\right)\right)}{1 \leq i_{1}<\cdots<i_{k} \leq n j \prod_{j=1}^{k} w_{i_{j}}}\right)^{1 / k}
\end{aligned}
$$

Also, since $T_{x_{i}} \geq T_{x_{i}}^{\prime}$, it can be obtained that:

$$
1-\prod_{j=1}^{k} T_{x_{i_{j}}} \leq 1-\prod_{j=1}^{k} T_{x_{i_{j}}^{\prime}}
$$

Further, we can achieve the equation shown in Box V.

Consequently, $T \geq T^{\prime}$. Similarly, we can have $I \leq I^{\prime}$ and $F \leq \overline{F^{\prime}}$. In the end, we have $<$ $s_{\theta},(T, I, F)>\geq<s_{\theta^{\prime}},\left(T^{\prime}, I^{\prime}, F^{\prime}\right)>$. That is to say:

$$
\begin{aligned}
& \operatorname{SVNLRWMSM}^{(k)}\left(x_{1}, x_{2}, \cdots, x_{n}\right) \geq \\
& \operatorname{SVNLRWMSM}^{(k)}\left(x_{1}^{\prime}, x_{2}^{\prime}, \cdots, x_{n}^{\prime}\right) .
\end{aligned}
$$

Theorem 3 (Commutativity). Let $\left(x_{1}^{\prime}, x_{2}^{\prime}, \cdots, x_{n}^{\prime}\right)$ be any permutation of $\left(x_{1}, x_{2}, \cdots, x_{n}\right)$, then:

$$
\begin{aligned}
& \operatorname{SVNLRWMSM}^{(k)}\left(x_{1}, x_{2}, \cdots, x_{n}\right)= \\
& \operatorname{SVNLRWMSM}^{(k)}\left(x_{1}^{\prime}, x_{2}^{\prime}, \cdots, x_{n}^{\prime}\right) .
\end{aligned}
$$

\section{Proof:}

$$
\begin{aligned}
& \operatorname{SVNLRWMSM}^{(k)}\left(x_{1}, x_{2}, \cdots, x_{n}\right)= \\
& \left.\qquad \frac{\sum_{1 \leq i_{1}<\cdots<i_{k} \leq n}\left(\prod_{j=1}^{k} w_{i_{j}}\right)\left(\prod_{j=1}^{k} x_{i_{j}}\right)}{\sum_{1 \leq i_{1}<\cdots<i_{k} \leq n} \prod_{j=1}^{k} w_{i_{j}}}\right)^{1 / k} \\
& =\left(\begin{array}{c}
\sum_{1 \leq i_{1}<\cdots<i_{k} \leq n}\left(\prod_{j=1}^{k} w_{i_{j}}\right)\left(\prod_{j=1}^{k} x_{i_{j}}\right) \\
\sum_{1 \leq i_{1}<\cdots<i_{k} \leq n} \prod_{j=1}^{k} w_{i_{j}}^{\prime}
\end{array}\right)^{1 / k} \\
& =\operatorname{SVNLRWMSM}^{(k)}\left(x_{1}^{\prime}, x_{2}^{\prime}, \cdots, x_{n}^{\prime}\right) .
\end{aligned}
$$

Theorem 4 (Idempotency). Let $x_{i}(i=1,2, \cdots, n)$ be a set of $S V N L N s$; if $x_{i}=x=<s_{\theta},(T, I, F)>$ for $\forall i$, then:

$$
\operatorname{SVNLRWMSM}^{(k)}\left(x_{1}, x_{2}, \cdots, x_{n}\right)=x .
$$




$$
\begin{aligned}
& \operatorname{SVNLRWMSM}^{(k)}\left(x_{1}, x_{2}, \cdots, x_{n}\right)=\left(\frac{\sum_{1 \leq i_{1}<\cdots<i_{k} \leq n}\left(\prod_{j=1}^{k} w_{i_{j}}\right)\left(\prod_{j=1}^{k} x_{i_{j}}\right)}{\sum_{1 \leq i_{1}<\cdots<i_{k} \leq n} \prod_{j=1}^{k} w_{i_{j}}}\right)^{1 / k} \\
& =\left\langle s\left(\frac{\underset{1 \leq i_{1}<\cdots<i_{k} \leq n}{\sum}\left(\prod_{j=1}^{k} w_{i_{j}}\right)\left(\prod_{j=1}^{k} \theta\left(x_{i_{j}}\right)\right)}{1 \leq i_{1}<\cdots<i_{k} \leq n j \prod_{j=1}^{k} w_{i_{j}}}\right)^{1 / k},\right. \\
& \left(\left(1-\left(\prod_{1 \leq i_{1}<\cdots<i_{k} \leq n}\left(1-\prod_{j=1}^{k} T_{x_{i_{j}}}\right)^{\prod_{j=1}^{k} w_{i_{j}}}\right)^{\frac{1}{1 \leq i_{1}<\cdots<i_{k} \leq n j \prod_{j=1}^{k} w_{i_{j}}}}\right)^{1 / k},\right. \\
& 1-\left(1-\left(\prod_{1 \leq i_{1}<\cdots<i_{k} \leq n}\left(1-\prod_{j=1}^{k}\left(1-I_{x_{i_{j}}}\right)\right)^{\prod_{j=1}^{k} w_{i_{j}}}\right)^{\frac{1}{1 \leq i_{1}<\cdots<i_{k} \leq n j=1} \prod_{j=1}^{k} w_{i_{j}}}\right)^{1 / k}, \\
& \left.\left.1-\left(1-\left(\prod_{1 \leq i_{1}<\cdots<i_{k} \leq n}\left(1-\prod_{j=1}^{k}\left(1-F_{x_{i_{j}}}\right)\right)^{\prod_{j=1}^{k} w_{i_{j}}}\right)^{\frac{1}{1 \leq i_{1}<\cdots<i_{k} \leq n j=1} \prod^{k} w_{i_{j}}}\right)^{1 / k}\right)\right\rangle .
\end{aligned}
$$

Box III

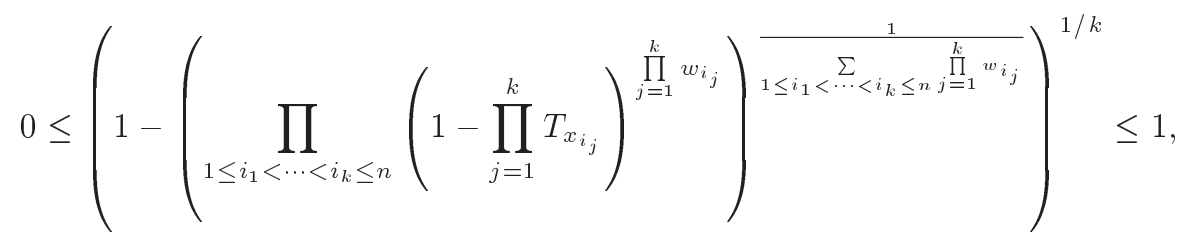

$0 \leq 1-\left(1-\left(\prod_{1 \leq i_{1}<\cdots<i_{k} \leq n}\left(1-\prod_{j=1}^{k}\left(1-I_{x_{i_{j}}}\right)\right)^{\prod_{j=1}^{k} w_{i_{j}}}\right)^{\frac{1}{1 \leq i_{1}<\cdots<i_{k} \leq n j=1} \sum^{\sum} w_{i_{j}}}\right)^{1 / k} \leq 1$,

$0 \leq 1-\left(1-\left(\prod_{1 \leq i_{1}<\cdots<i_{k} \leq n}\left(1-\prod_{j=1}^{k}\left(1-F_{x_{i_{j}}}\right)\right)^{\prod_{j=1}^{k} w_{i_{j}}}\right)^{\frac{1}{1 \leq i_{1}<\cdots<i_{k} \leq n j \prod_{j}^{k} w_{i_{j}}}}\right)^{1 / k} \leq 1$ 


$$
\begin{aligned}
& \left(1-\left(\prod_{1 \leq i_{1}<\cdots<i_{k} \leq n}\left(1-\prod_{j=1}^{k} T_{x_{i_{j}}}\right)^{\prod_{j=1}^{k} w_{i_{j}}}\right)^{\frac{1}{1 \leq i_{1}<\sum_{<i_{k} \leq n} \prod_{j=1}^{k} w_{i_{j}}}}\right)^{1 / k} \geq \\
& \left(1-\left(\prod_{1 \leq i_{1}<\cdots<i_{k} \leq n}\left(1-\prod_{j=1}^{k} T_{x_{i_{j}}^{\prime}}\right)^{\prod_{j=1}^{k} w_{i_{j}}}\right)^{\frac{1}{1 \leq i_{1}<\cdots<i_{k} \leq n}{ }^{\sum} \prod_{j=1}^{k} w_{i_{j}}}\right)^{1 / k} .
\end{aligned}
$$

Box V

\section{Proof:}

$$
\begin{aligned}
& \text { SVNLRWMSM }^{(k)}\left(x_{1}, x_{2}, \cdots, x_{n}\right) \\
& =\left(\frac{\sum_{1 \leq i_{1}<\cdots<i_{k} \leq n}\left(\prod_{j=1}^{k} w_{i_{j}}\right)\left(\prod_{j=1}^{k} x_{i_{j}}\right)}{\sum_{1 \leq i_{1}<\cdots<i_{k} \leq n} \prod_{j=1}^{k} w_{i_{j}}}\right)^{1 / k} \\
& =\left(\frac{\sum_{1 \leq i_{1}<\cdots<i_{k} \leq n}\left(\prod_{j=1}^{k} w_{i_{j}}\right)\left(\prod_{j=1}^{k} x\right)}{\sum_{1 \leq i_{1}<\cdots<i_{k} \leq n} \prod_{j=1}^{k} w_{i_{j}}}\right)^{1 / k} \\
& =\left(\frac{\sum_{1 \leq i_{1}<\cdots<i_{k} \leq n} \prod_{j=1}^{k} w_{i_{j}}}{\sum_{1 \leq i_{1}<\cdots<i_{k} \leq n} \prod_{j=1}^{k} w_{i_{j}}}\right)^{1 / k}=x .
\end{aligned}
$$

Theorem 5 (Boundedness). Let $x_{i}(i=1,2, \cdots, n)$ be a set of SVNLNs, and:

$$
\begin{aligned}
& x^{+}=<s_{\max _{i=1}^{n} \theta_{i}},\left(\max _{i=1}^{n} T_{i}, \min _{i=1}^{n} I_{i}, \min _{i=1}^{n} F_{i}\right)>, \\
& x^{-}=<s_{\min _{i=1}^{n} \theta_{i}},\left(\min _{i=1}^{n} T_{i}, \max _{i=1}^{n} I_{i}, \max _{i=1}^{n} F_{i}\right)>,
\end{aligned}
$$
then:

$$
x^{-} \leq \operatorname{SVNLRWMSM}^{(k)}\left(x_{1}, x_{2}, \cdots, x_{n}\right) \leq x^{+} .
$$

Proof. According to the idempotency and monotonicity, we can obtain:

$$
\begin{aligned}
& \operatorname{SVNLRWMSM}^{(k)}\left(x_{1}, x_{2}, \cdots, x_{n}\right) \leq \\
& \operatorname{SVNLRWMSM}^{(k)}\left(x^{+}, x^{+}, \cdots, x^{+}\right) \text {and } \\
& \operatorname{SVNLRWMSM}^{(k)}\left(x_{1}, x_{2}, \cdots, x_{n}\right) \geq \\
& \operatorname{SVNLRWMSM}^{(k)}\left(x^{-}, x^{-}, \cdots, x^{-}\right) .
\end{aligned}
$$

Hence, we have:

$$
x^{-} \leq \operatorname{SVNLRWMSM}^{(k)}\left(x_{1}, x_{2}, \cdots, x_{n}\right) \leq x^{+} .
$$

Next, we discuss two particular cases of the SVNLRWMSM by regulating the argument $k$ :

Case 1: In the event of $k=1$, the SVNLRWMSM degenerates into an SVNLWA operator [16].

$$
\begin{aligned}
& \operatorname{SVNLRWMSM}^{(1)}\left(x_{1}, x_{2}, \cdots, x_{n}\right) \\
&=\left\langle s \sum_{1 \leq i_{1} \leq n} w_{i} \theta\left(x_{i_{1}}\right),\left(1-\prod_{1 \leq i_{1} \leq n}\left(1-T_{x_{i_{1}}}\right)^{w_{i_{1}}},\right.\right. \\
&\left.\left.\prod_{1 \leq i_{1} \leq n} I_{x_{i_{1}}}^{w_{i_{1}}}, \prod_{1 \leq i_{1} \leq n} F_{x_{i_{1}}}^{w_{i_{1}}}\right)\right\rangle \\
&= \operatorname{SVNLWA}\left(x_{1}, x_{2}, \cdots, x_{n}\right) .
\end{aligned}
$$

Case 2: In the event of $k=n$, the SVNLRWMSM degenerates into an Single-Valued Neutrosophic Linguistic Geometric (SVNLG) operator.

$$
\begin{aligned}
& \operatorname{SVNLRWMSM}^{(n)}\left(x_{1}, x_{2}, \cdots, x_{n}\right)= \\
& =\left\langle s \prod_{j=1}^{n} \theta^{\frac{1}{n}}\left(x_{i_{j}}\right)\right. \\
& \\
& \left.\left.\quad \prod_{1 \leq i_{1} \leq n} I_{x_{i_{1}}}^{\frac{1}{n}}, \prod_{1 \leq i_{1} \leq n} F_{x_{i_{1}}}^{\frac{1}{n}}\right)\right\rangle \\
& =\operatorname{SVNLG}\left(1-\prod_{1}, x_{2}, \cdots, x_{n}\right) .
\end{aligned}
$$

\subsection{SVNLRWDMSM operator}

Definition 9. Let $x_{i}=<s_{\theta\left(x_{i}\right)},\left(T_{x_{i}}, I_{x_{i}}, F_{x_{i}}\right)>$ $(i=1,2, \cdots, n)$ be a set of SVNLNs and let $W=$ 
$\left(w_{1}, w_{2}, \cdots, w_{n}\right)^{T}$ be the weight vector in which $w_{i} \in$ $[0,1]$ and $\sum_{i=1}^{n} w_{i}=1$. The SVNLRWDMSM, $\Omega^{n} \rightarrow$ $\Omega$; an SVNLRWDMSM operator is suggested in the following:

$$
\begin{aligned}
& \operatorname{SVNLRWDMSM}^{(k)}\left(x_{1}, x_{2}, \cdots, x_{n}\right)= \\
& \frac{\prod_{\prod_{i}<\cdots<i_{k} \leq n}\left(\sum_{j=1}^{k} x_{i_{j}}\right) \frac{\sum_{j=1}^{k} w_{i j}}{1 \leq i_{1}<\cdots<i_{k} \leq n} \sum_{j=1}^{k} w_{i_{j}}}{k},
\end{aligned}
$$

where $\Omega$ is the set of entire SVNLNs and then, SVNLRWDMSM is known as the single-valued neutrosophic linguistic RWDMSM operator.

Based on the operational laws of the SVNLNs defined in Definition 2 and Eq. (12), we can obtain the aggregated results shown in Theorem 6 .

Theorem 6. Let $x_{i}=<s_{\theta\left(x_{i}\right)},\left(T_{x_{i}}, I_{x_{i}}, F_{x_{i}}\right)>$ $(i=1,2, \cdots, n)$ be a set of $S V N L N s$, and let $W=$ $\left(w_{1}, w_{2}, \cdots, w_{n}\right)^{T}$ be the weight vector with $w_{i} \in[0,1]$ and $\sum_{i=1}^{n} w_{i}=1$; then, the result of SVNLRWDMSM operator is notwithstanding an SVNLN.

Eq. (13) is shown in Box VI.
Remark 1. The SVNLRWDMSM operator also owns the monotonicity, idempotency, boundedness, and commutativity.

Later, we discuss some particular cases of the SVNLRWDMSM by regulating the argument $k$.

Case 1: In the event of $k=1$, the SVNLRWDMSM reduces to an SVNLWG operator.

$$
\begin{aligned}
& \operatorname{SVNLRWDMSM}^{(1)}\left(x_{1}, x_{2}, \cdots, x_{n}\right) \\
& =<s \prod_{1 \leq i_{1} \leq n} \theta^{w_{i_{j}}}\left(x_{i_{j}}\right),\left(\prod_{1 \leq i_{1} \leq n} T_{x_{i_{j}}}^{w_{i_{j}}},\right. \\
& \quad \prod_{1 \leq i_{1} \leq n} 1-\left(1-I_{x_{i_{j}}}\right)^{w_{i_{j}}}, \\
& \left.\prod_{1 \leq i_{1} \leq n} 1-\left(1-F_{x_{i_{j}}}\right)^{w_{i_{j}}}\right)> \\
& =\operatorname{SVNLWG}\left(x_{1}, x_{2}, \cdots, x_{n}\right) .
\end{aligned}
$$

Case 2: In the event of $k=n$, the SVNLRWDMSM

$$
\begin{aligned}
& \operatorname{SVNLRWDMSM}^{(k)}\left(x_{1}, x_{2}, \cdots, x_{n}\right) \\
& =\langle s \\
& \underbrace{\prod_{j=1}\left(\sum_{j=1}^{k} \theta\left(x_{i_{j}}\right)\right) \sum_{j=1}^{\sum_{1 \leq i_{1}<\cdots<i_{k} \leq n} w_{i_{j}}}}_{1 \leq i_{1}<\cdots<i_{k} \leq n} \\
& \left(1-\left(1-\left(\prod_{1 \leq i_{1}<\cdots<i_{k} \leq n}\left(1-\prod_{j=1}^{k}\left(1-T_{x_{i_{j}}}\right)\right)^{\sum_{j=1}^{k} w_{i_{j}}}\right)^{\frac{1}{1 \leq i_{1}<\cdots<i_{k} \leq n} \sum_{j=1}^{\sum_{j}^{k} w_{i}}}\right)^{1 / k},\right. \\
& \left(1-\left(\prod_{1 \leq i_{1}<\cdots<i_{k} \leq n}\left(1-\prod_{j=1}^{k} I_{x_{i_{j}}}\right)^{\sum_{j=1}^{k} w_{i_{j}}}\right)^{\frac{1}{1 \leq i_{1}<\cdots<i_{k} \leq n} \sum_{j=1}^{\sum_{j=1}^{k} w_{i_{j}}}}\right)^{1 / k}, \\
& \left.\left.\left(1-\left(\prod_{1 \leq i_{1}<\cdots<i_{k} \leq n}\left(1-\prod_{j=1}^{k} F_{x_{i_{j}}}\right)^{\sum_{j=1}^{k} w_{i_{j}}}\right)^{\frac{1}{1 \leq i_{1}<\cdots<i_{k} \leq n} \sum_{j=1}^{\sum_{j=1}^{k} w_{i}}}\right)^{1 / k}\right)\right\rangle .
\end{aligned}
$$


reduces to an SVNLA operator [16].

$$
\begin{aligned}
& \operatorname{SVNLRWMSM}^{(n)}\left(x_{1}, x_{2}, \cdots, x_{n}\right)= \\
& =\left\langle s \sum_{j=1}^{n} \frac{\theta\left(x_{i_{j}}\right)}{n},\left(1-\prod_{1 \leq i_{1} \leq n}\left(1-T_{x_{i_{1}}}\right)^{\frac{1}{n}},\right.\right. \\
& \left.\left.\prod_{1 \leq i_{1} \leq n} I_{x_{i_{1}}}^{\frac{1}{n}}, \prod_{1 \leq i_{1} \leq n} F_{x_{i_{1}}}^{\frac{1}{n}}\right)\right\rangle \\
& =\operatorname{SVNLA}\left(x_{1}, x_{2}, \cdots, x_{n}\right)
\end{aligned}
$$

Remark 2. In particular, it is vital that SVNLRWMSM or SVNLRWDMSM operator cannot have the reciprocity among many given arguments while $k=1$ or $k=n$. In other words, both of them are reduced into the self-governed operators such as SVNLA, SVNLG, SVNWLA, and SVNWLG [16].

\section{MCDM algorithms based on SVNLRWMSM and SVNLRWDMSM}

\subsection{Description of the MCDM problems}

Suppose that $A=\left\{A_{1}, A_{2}, \cdots, A_{m}\right\}$ be a set of alternatives, $C=\left\{C_{1}, C_{2}, \cdots, C_{n}\right\}$ be a set of $n$ cri- teria, and $W=\left\{w_{1}, w_{2}, \cdots, w_{n}\right\}$ be the weight vector assigned to the attributes by the DMs with the normal constraints $w_{j} \in[0,1], \sum_{j=1}^{n} w_{j}=1$. Assume that the whole evaluation information of the alternatives with respect to criteria is suggested by SVNL matrix $P=\left(p_{i j}\right)_{m \times n}=\left(<s_{\theta_{i j}},\left(T_{i j}, I_{i j}, F_{i j}\right)>\right)_{m \times n}$. The evaluation values associated with the alternatives for the formalization of MCDM issues are given in Table 1.

\subsection{The MCDM method based on SVNLRWMSM or SVNLRWDMSM operator}

In order to make a decision, the decision-making framework for employing the proposed methods is presented in Figure 1.

In the meantime, Algorithm 1 is given for SVNLRWMSM or SVNLRWDMSM operator.

\subsection{A case of TMESQE}

In determining the TMESQE indicators, the literature research method is mainly adopted and the index system is constructed by summarizing relevant research results and combining practical investigation with index design principles. Upon taking all factors into consideration, the relevant literature is studied to

Table 1. A general Single-Valued Neutrosophic Linguistic (SVNL) Multi-Criteria Decision Making (MCDM) matrix.

\begin{tabular}{ccccc}
\hline & $\boldsymbol{C}_{\mathbf{1}}$ & $\boldsymbol{C}_{\mathbf{2}}$ & $\cdots$ & $\boldsymbol{C}_{\boldsymbol{n}}$ \\
\hline$A_{1}$ & $\left\langle s_{\theta_{11}},\left(T_{11}, I_{11}, F_{11}\right)>\right.$ & $<s_{\theta_{12}},\left(T_{12}, I_{12}, F_{12}\right)>$ & $\cdots$ & $<s_{\theta_{1 n}},\left(T_{1 n}, I_{1 n}, F_{1 n}\right)>$ \\
$A_{2}$ & $\left\langle s_{\theta_{21}},\left(T_{21}, I_{21}, F_{21}\right)\right\rangle$ & $<s_{\theta_{22}},\left(T_{22}, I_{22}, F_{22}\right)>$ & $\cdots$ & $<s_{\theta_{2 n}},\left(T_{2 n}, I_{2 n}, F_{2 n}\right)>$ \\
$\vdots$ & $\vdots$ & $\vdots$ & $\ddots$ & $\vdots$ \\
$A_{m}$ & $\left.<s_{\theta_{m 1}},\left(T_{m 1}, I_{m 1}, F_{m 1}\right)\right\rangle$ & $<s_{\theta_{m 2}},\left(T_{m 2}, I_{m 2}, F_{m 2}\right)>$ & $\cdots$ & $<s_{\theta_{m n}},\left(T_{m n}, I_{m n}, F_{m n}\right)>$ \\
\hline
\end{tabular}

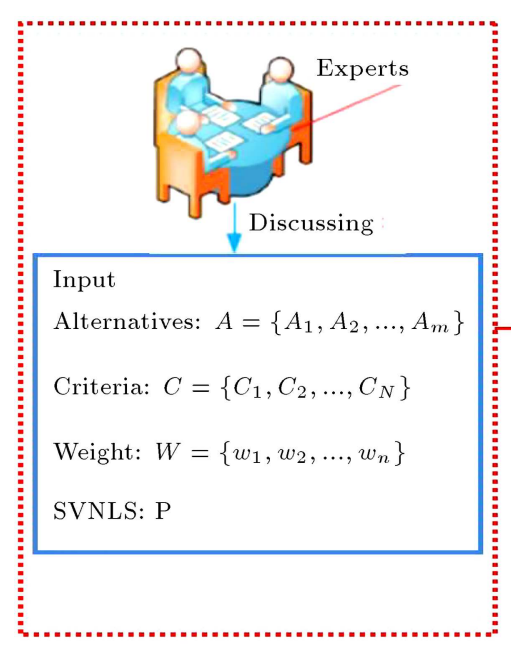

The MCDM issue

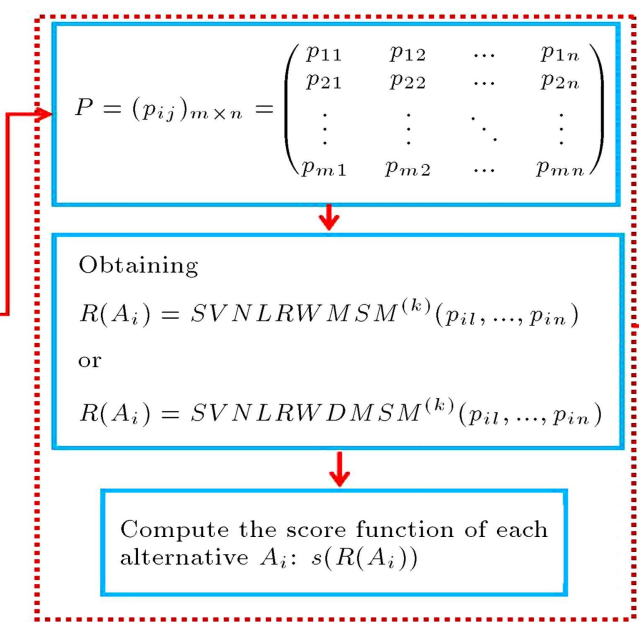

Collecting and handling experts' evaluation information
Achieving the final ranking order and output the optimal alternative

Figure 1. The decision-making framework for employing the developed methods. Single-Valued Neutrosophic Linguistic Set: SVNLS; Single-Valued Neutrosophic Linguistic Reducible Weighted Maclaurin Symmetric Mean: SVNLRWMSM; Single-Valued Neutrosophic Linguistic Reducible Weighted Dual Maclaurin Symmetric Mean: SVNLRWDMSM; and Multi-Criteria Decision-Making: MCDM. 


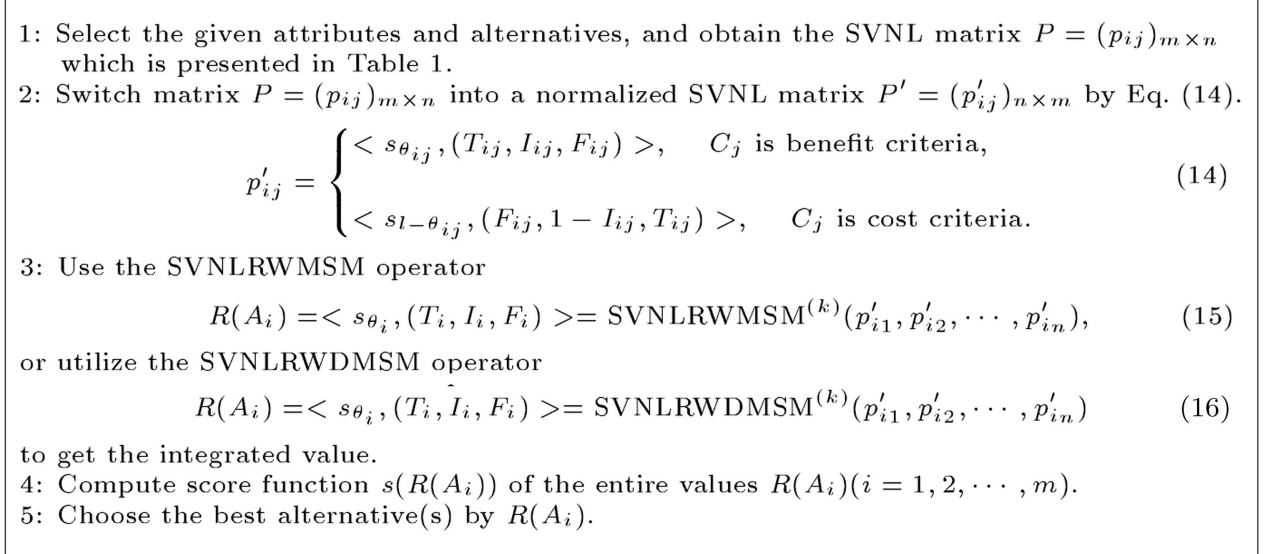

Algorithm 1. SVNLRWMSM or SVNLRWDMSM operator.

collect and sort out the previous influencing factors of user experience, tourism service, and mobile ecommerce. According to the above, six evaluation dimensions are extracted, which are content experience, interactivity, security, sensitivity, personalization, and travel experience.

A preliminary evaluation index system of TME service quality level is built. Six preliminary criteria $\left(C_{1}, C_{2}, C_{3}, C_{4}, C_{5}, C_{6}\right)$ are established in the evaluation index system related to tourism products. The detailed description of each criterion is given in Table 2 .

Example 1. Suppose that there are five influential tourism products $A=\left\{A_{1}, A_{2}, A_{3}, A_{4}, A_{5}\right\}$ to be considered. The experts choose the highly representative attribute sets:

- $C=\left\{C_{1}\right.$ (Content experience);

- $C_{2}$ (Interactivity);

- $C_{3}$ (Security);

- $C_{4}$ (Sensitivity);

- $C_{5}$ (Personalization);

- and $C_{6}$ (Travel experience)\}.

According to the evaluation principle of the TME service quality, we can determine that the whole criteria are benefit criteria. Moreover, the weight information given by experts is $w=(0.2,0.3,0.1,0.1,0.1,0.2)$. The experts assess these tourism products by SVNLNs under the LTS $S=\left\{s_{0}=\right.$ extremely bad, $s_{1}=$ very bad, $s_{2}=$ bad, $s_{3}=$ fair, $s_{4}=$ good, $s_{5}=$ very good, and $s_{6}=$ extremely good $\}$ and generate the final SVNL matrix in Table 3 .

Next, we employ the above developed algorithm in selecting the most popular tourism product under SVNL text.

Step 1: Select the attributes and alternatives and achieve the SVNL matrix $P=\left(p_{i j}\right)_{5 \times 6}$ which is presented in Table 3.

Step 2: No transformation is needed because entire criteria are beneficial criteria.

Step 3: Utilize the SVNLRWMSM ${ }^{(1)}$ operator to integrate the decision value as follows:

$$
\begin{aligned}
& R\left(A_{1}\right)=<s_{5.2000},(0.8375,0.1072,0.1231)> \\
& R\left(A_{2}\right)=<s_{4.7000},(0.7509,0.1741,0.1692)> \\
& R\left(A_{3}\right)=<s_{4.3000},(0.6768,0.1741,0.2195)> \\
& R\left(A_{4}\right)=<s_{3.7000},(0.5655,0.1888,0.1911)> \\
& R\left(A_{5}\right)=<s_{3.4000},(0.4644,0.1888,0.1911)>
\end{aligned}
$$

or utilize SVNLRWDMSM ${ }^{(1)}$ operator to integrate the decision value as follows:

$$
\begin{aligned}
& R\left(A_{1}\right)=<s_{5.1857},(0.8288,0.1105,0.1312)> \\
& R\left(A_{2}\right)=<s_{4.6382},(0.7354,0.1809,0.1822)> \\
& R\left(A_{3}\right)=<s_{4.2149},(0.6670,0.1809,0.2326)> \\
& R\left(A_{4}\right)=<s_{3.6222},(0.5562,0.2025,0.2143)> \\
& R\left(A_{5}\right)=<s_{3.3227},(0.4554,0.2025,0.2143)>
\end{aligned}
$$

Step 4: Calculate the score function $s\left(R\left(A_{i}\right)\right)$ of the entire values $R\left(A_{i}\right)(i=1,2,3,4,5)$ as follows:

\section{SVNLRWMSM:}

$$
\begin{array}{ll}
s\left(R\left(A_{1}\right)\right)=4.5192, & s\left(R\left(A_{2}\right)\right)=3.7719, \\
s\left(R\left(A_{3}\right)\right)=3.2726, & s\left(R\left(A_{4}\right)\right)=2.6956, \\
s\left(R\left(A_{5}\right)\right)=2.3624 . &
\end{array}
$$


Table 2. The evaluation criteria of tourism products.

\begin{tabular}{|c|c|}
\hline Criteria & Brief description \\
\hline Content experience $\left(C_{1}\right)$ & $\begin{array}{l}\text { TME has very rich service functions. Users can query tourism } \\
\text { information, payment, booking and sharing experience through the service platform on } \\
\text { mobile devices. All the service functions of TME are designed on the basis of } \\
\text { content; thus, the quality of service content is closely related to the } \\
\text { quality of user experience. By sorting out the literature, the content } \\
\text { perception from five aspects, including complete and comprehensive services, } \\
\text { true and accurate information content, novel and timely information content, } \\
\text { easy to understand in information and concise and orderly information content. }\end{array}$ \\
\hline Interactivity $\left(C_{2}\right)$ & $\begin{array}{l}\text { The real purpose of user experience is to get information content, and the main } \\
\text { means is to use interaction, which can ensure the smooth process of user's content } \\
\text { experience. The interaction behavior appears in the whole process of user } \\
\text { experience. The interaction process between users and products is the learning } \\
\text { process in which users are gradually becoming familiar with service products. Each step of } \\
\text { user expansion of service products can be regarded as the communication and } \\
\text { interaction with service products. Interactivity can be reflected in the following } \\
\text { five aspects: easy to learn service products, smooth operation process, } \\
\text { good fault tolerance, timely feedback and real-time interaction. }\end{array}$ \\
\hline Security $\left(C_{3}\right)$ & $\begin{array}{l}\text { As is known, the basic principle of mobile online travel is absolute safety. } \\
\text { For example, when booking and paying for tickets or tickets for } \\
\text { scenic spots, some users will also consider the validity and security of } \\
\text { the transaction and worry about whether their bank and private information } \\
\text { will be leaked. Therefore, before providing the service, travel mobile } \\
\text { e-commerce service operators should solve the security problems that consumers } \\
\text { are worried about and guarantee that they can enjoy the best service. } \\
\text { Security can be reflected in four aspects: payment security, transaction } \\
\text { validity, protection of rights and interests, and data information security. }\end{array}$ \\
\hline Personalization $\left(C_{5}\right)$ & $\begin{array}{l}\text { Different users have different demands and expectations for service information. } \\
\text { Even the functional preferences and interaction behaviors of the same } \\
\text { service product may have different requirements due to individual differences; } \\
\text { thus, the experiential experience in the service process will be different. } \\
\text { When providing mobile tourism services for users, individual differences } \\
\text { should be taken into account, including the differences between different } \\
\text { individuals in their hobbies and content needs. Based on this, the function of } \\
\text { online travel service is adjusted to meet the diverse standards of different } \\
\text { users. It can be reflected in four aspects: conforming to user habits, } \\
\text { meeting user preferences and expectations, upgrading and updating of service } \\
\text { products, and emotional perception of users. }\end{array}$ \\
\hline Travel experience $\left(C_{6}\right)$ & $\begin{array}{l}\text { As the key content of online travel, travel experience is of great significance. } \\
\text { Even if customers cannot experience good e-services on the platform and } \\
\text { if they can create perfect practical experience for users, the overall } \\
\text { service quality will be highly evaluated. It is reflected as follows: } \\
\text { real product information description, service attitude, etiquette in place, } \\
\text { and service emergency remedy. }\end{array}$ \\
\hline
\end{tabular}


Table 3. The Single-Valued Neutrosophic Linguistic (SVNL) matrix in Example 1.

\begin{tabular}{|c|c|c|c|}
\hline & $C_{1}$ & $C_{2}$ & $C_{3}$ \\
\hline$A_{1}$ & $<s_{6},(0.9,0.1,0.1)>$ & $<s_{5},(0.8,0.1,0.2)>$ & $<s_{5},(0.8,0.2,0.1)>$ \\
\hline$A_{2}$ & $<s_{6},(0.8,0.1,0.2)>$ & $<s_{5},(0.8,0.2,0.2)>$ & $<s_{4},(0.7,0.2,0.3)>$ \\
\hline$A_{3}$ & $<s_{6},(0.7,0.1,0.2)>$ & $<s_{4},(0.7,0.2,0.3)>$ & $<s_{3},(0.6,0.2,0.1)>$ \\
\hline$A_{4}$ & $<s_{5},(0.5,0.1,0.2)>$ & $<s_{4},(0.6,0.2,0.3)>$ & $<s_{3},(0.6,0.2,0.2)>$ \\
\hline \multirow[t]{2}{*}{$A_{5}$} & $<s_{5},(0.4,0.1,0.2)>$ & $<s_{3},(0.5,0.2,0.3)>$ & $<s_{3},(0.5,0.2,0.2)>$ \\
\hline & $C_{4}$ & $C_{5}$ & $C_{6}$ \\
\hline$A_{1}$ & $<s_{5},(0.9,0.1,0.1)>$ & $<s_{5},(0.8,0.1,0.1)>$ & $<s_{5},(0.8,0.1,0.1)>$ \\
\hline$A_{2}$ & $<s_{4},(0.8,0.2,0.2)>$ & $<s_{4},(0.7,0.2,0.1)>$ & $<s_{4},(0.6,0.2,0.1)>$ \\
\hline$A_{3}$ & $<s_{4},(0.8,0.2,0.3)>$ & $<s_{4},(0.6,0.2,0.2)>$ & $<s_{4},(0.6,0.2,0.2)>$ \\
\hline$A_{4}$ & $<s_{3},(0.7,0.2,0.3)>$ & $<s_{3},(0.5,0.2,0.1)>$ & $<s_{3},(0.5,0.3,0.1)>$ \\
\hline$A_{5}$ & $<s_{3},(0.6,0.2,0.3)>$ & $<s_{3},(0.4,0.2,0.1)>$ & $<s_{3},(0.4,0.3,0.1)>$ \\
\hline
\end{tabular}

\section{SVNLRWDMSM:}

$$
\begin{array}{ll}
s\left(R\left(A_{1}\right)\right)=4.4718, & s\left(R\left(A_{2}\right)\right)=3.6676, \\
s\left(R\left(A_{3}\right)\right)=3.1660, & s\left(R\left(A_{4}\right)\right)=2.5831, \\
s\left(R\left(A_{5}\right)\right)=2.2579 . &
\end{array}
$$

Step 5: According to the above score function $s\left(R\left(A_{i}\right)\right)(i=1,2,3,4,5)$, we can achieve the ranking of the tourism products as $A_{1} \succ A_{2} \succ A_{3} \succ A_{4} \succ A_{5}$.

\subsection{Sensitivity analysis}

For discussing the sensitivity of the parameters $k$ of score values, an experiment (Example 1) was constructed by taking various values of $k(k=$ $1,2,3,4,5,6)$.

According to the SVNLRWMSM operator and SVNLRWDMSM operator, the final score values of five tourism products are shown in Figure 2 and Table 4, respectively. From Figure 2 and Table 4, some key points are given in the following:

1. For SVNLRWMSM, the score values of the entire five tourism products are monotonically increasing first if $k \in[1,5]$ and later monotonically decreasing if $k \in[5,6]$. Moreover, it is not difficult to determine the final ranking owing to the same values obtained that vary from 2.2396 to 4.4623 with a diverse value of 2.22 when $k=6$. The final ranking results are all ranked as:

$$
A_{1} \succ A_{2} \succ A_{3} \succ A_{4} \succ A_{5} .
$$

2. For SVNLRWDMSM, the score values of the entire five tourism products are firstly monotonically decreasing if $k \in[1,5]$ and later monotonically increasing if $k \in[5,6]$. In addition, it is very clear to determine the final ranking compared with the

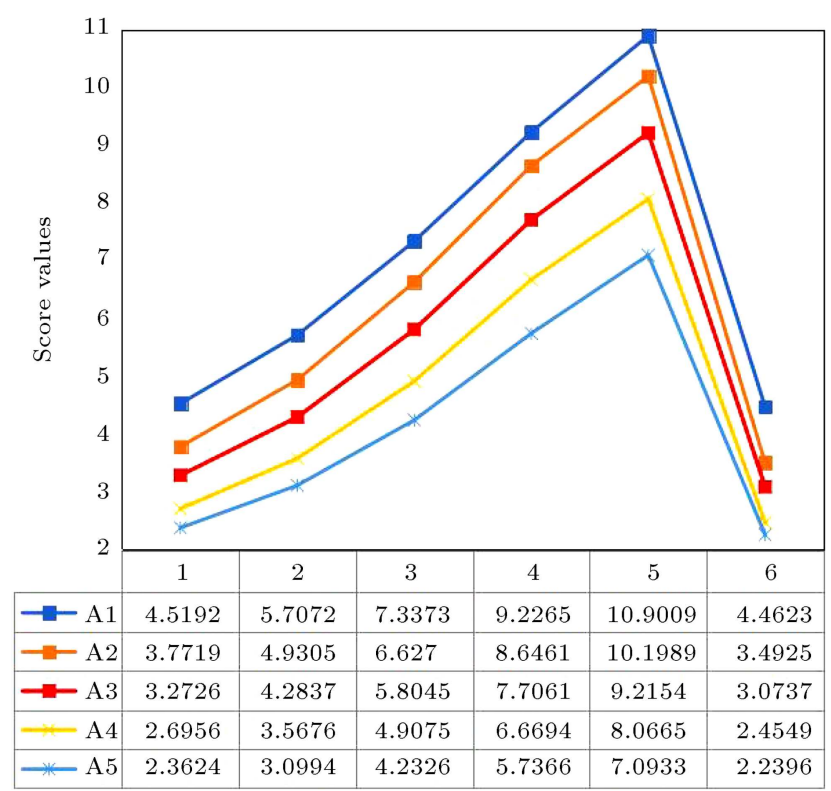

Figure 2. The total changing trend of parameter $k$ based on Single-Valued Neutrosophic Linguistic Reducible Weighted MSM (SVNLRWMSM).

SVNLRWMSM algorithm when $k=3,4,5$. The final results are all ranked as follows:

$$
A_{1} \succ A_{2} \succ A_{3} \succ A_{4} \succ A_{5}
$$

3. The reason for the inflection point is that $k=1$ is the modality of AO firstly and $k=6$ is the modality of Geometric Operator (GO) at the end for SVNLRWMSM. Homoplastically, it experiences the transformation from GO to AO for SVNLRWDMSM.

4. The values at both ends $(k=1$ and $k=6)$ have an interesting case. For SVNLRWMSM, the score of five tourism products is the minimum compared with $k=1,2,3,4,5$ if $k=6$ (GO form). For 
Table 4. Ranking results of the tourism products with different k's by Single-Valued Neutrosophic Linguistic Reducible Weighted Dual MSM (SVNLRWDMSM) algorithm.

\begin{tabular}{|c|c|c|c|}
\hline$k$ & Score values & Ordering & Optimal product \\
\hline$k=1$ & $\begin{array}{l}s\left(R\left(A_{1}\right)\right)=4.4718 \\
s\left(R\left(A_{2}\right)\right)=3.6676 \\
s\left(R\left(A_{3}\right)\right)=3.1660 \\
s\left(R\left(A_{4}\right)\right)=2.5831 \\
s\left(R\left(A_{5}\right)\right)=2.2579\end{array}$ & $A_{1} \succ A_{2} \succ A_{3} \succ A_{4} \succ A_{5}$ & $A_{1}$ \\
\hline$k=2$ & $\begin{array}{l}s\left(R\left(A_{1}\right)\right)=9.2555 \\
s\left(R\left(A_{2}\right)\right)=7.1671 \\
s\left(R\left(A_{3}\right)\right)=5.9762 \\
s\left(R\left(A_{4}\right)\right)=4.6179 \\
s\left(R\left(A_{5}\right)\right)=3.9624\end{array}$ & $A_{1} \succ A_{2} \succ A_{3} \succ A_{4} \succ A_{5}$ & $A_{1}$ \\
\hline$k=3$ & $\begin{array}{l}s\left(R\left(A_{1}\right)\right)=193.3486 \\
s\left(R\left(A_{2}\right)\right)=130.0276 \\
s\left(R\left(A_{3}\right)\right)=96.3677 \\
s\left(R\left(A_{4}\right)\right)=63.0655 \\
s\left(R\left(A_{5}\right)\right)=49.5881\end{array}$ & $A_{1} \succ A_{2} \succ A_{3} \succ A_{4} \succ A_{5}$ & $A_{1}$ \\
\hline$k=4$ & $\begin{array}{l}s\left(R\left(A_{1}\right)\right)=23854335.1334 \\
s\left(R\left(A_{2}\right)\right)=10580324.2317 \\
s\left(R\left(A_{3}\right)\right)=5179747.5209 \\
s\left(R\left(A_{4}\right)\right)=2014156.7743 \\
s\left(R\left(A_{5}\right)\right)=1166101.9310\end{array}$ & $A_{1} \succ A_{2} \succ A_{3} \succ A_{4} \succ A_{5}$ & $A_{1}$ \\
\hline$k=5$ & $\begin{array}{l}s\left(R\left(A_{1}\right)\right)=70631993944989128000000 \\
s\left(R\left(A_{2}\right)\right)=12635422385855326000000 \\
s\left(R\left(A_{3}\right)\right)=2058417037824523000000 \\
s\left(R\left(A_{4}\right)\right)=199406516030728930000 \\
s\left(R\left(A_{5}\right)\right)=45390641943246275000\end{array}$ & $A_{1} \succ A_{2} \succ A_{3} \succ A_{4} \succ A_{5}$ & $A_{1}$ \\
\hline$k=6$ & $\begin{array}{l}s\left(R\left(A_{1}\right)\right)=4.5059 \\
s\left(R\left(A_{2}\right)\right)=3.5925 \\
s\left(R\left(A_{3}\right)\right)=3.1862 \\
s\left(R\left(A_{4}\right)\right)=2.5683 \\
s\left(R\left(A_{5}\right)\right)=2.3333\end{array}$ & $A_{1} \succ A_{2} \succ A_{3} \succ A_{4} \succ A_{5}$ & $A_{1}$ \\
\hline
\end{tabular}

SVNLRWDMSM, the score of five tourism products is at a maximum compared with $k=1,2,3,4,5$ if $k=6$ (AO form).

\section{Comparative analysis}

In the following, some existing decision-making methods $[5,8,9,12-18]$ with their final decision-making results and their characteristics are explored in detail.

Remark 3. Based on Table 5, we can find that the developed algorithms have achieved the identical ordering results with the existing methods $[5,8,9,12,14-18]$, suggesting that the developed algorithms (SVNLRWMSM and SVNLRWDMSM) are feasible and reasonable for decision-making issues involving SVNL information.

With respect to some SVNL AOs, only the references $[15,18]$ took the reciprocity of the criteria into consideration. To better distinguish the features of existing SVNL AOs, we make an overview of them shown in Table 6. According to Table 6, the developed AOs are based on RWMSM operators with the parameter $k$. Therefore, the initiated AOs (SVNLRWMSM and SVNLRWDMSM) are more global than some existing 
Table 5. A comparison study in Example 1.

\begin{tabular}{lll}
\hline Algorithms & \multicolumn{1}{c}{ Ranking } & Optimal alternative \\
\hline Algorithm 1: SVNLRWMSM & $A_{1} \succ A_{2} \succ A_{3} \succ A_{4} \succ A_{5}$ & $A_{1}$ \\
Algorithm 1: SVNLRWDMSM & $A_{1} \succ A_{2} \succ A_{3} \succ A_{4} \succ A_{5}$ & $A_{1}$ \\
Ye [5] & $A_{1} \succ A_{2} \succ A_{3} \succ A_{4} \succ A_{5}$ & $A_{1}$ \\
Tian et al. [15] & $A_{1} \succ A_{2} \succ A_{3} \succ A_{4} \succ A_{5}$ & $A_{1}$ \\
Tian et al. [14] & $A_{1} \succ A_{2} \succ A_{3} \succ A_{4} \succ A_{5}$ & $A_{1}$ \\
Wang et al. [17] & $A_{1} \succ A_{2} \succ A_{3} \succ A_{4} \succ A_{5}$ & $A_{1}$ \\
Ji et al. [9] & $A_{1} \succ A_{2} \succ A_{3} \succ A_{4} \succ A_{5}$ & $A_{1}$ \\
Chen el al. [8] & $A_{1} \succ A_{2} \succ A_{3} \succ A_{4} \succ A_{5}$ & $A_{1}$ \\
Tian et al. [12] & $A_{1} \succ A_{2} \succ A_{3} \succ A_{4} \succ A_{5}$ & $A_{1}$ \\
Luo et al. [10] & $A_{1} \succ A_{2} \succ A_{3} \succ A_{4} \succ A_{5}$ & $A_{1}$ \\
Tan et al. [16] & $A_{1} \succ A_{2} \succ A_{3} \succ A_{4} \succ A_{5}$ & $A_{1}$ \\
Wang et al. [18] & $A_{1} \succ A_{2} \succ A_{3} \succ A_{4} \succ A_{5}$ & $A_{1}$ \\
Wang et al. [18] & $A_{1} \succ A_{2} \succ A_{3} \succ A_{4} \succ A_{5}$ & $A_{1}$ \\
Wang et al. [18] & $A_{1} \succ A_{2} \succ A_{3} \succ A_{4} \succ A_{5}$ & $A_{1}$ \\
& & \\
\hline aSVNLRWMSM: Single-Valued Neutrosophic Linguistic Reducible Weighted Maclaurin Symmetric Mean; \\
bSVNLRWDMSM: Single-Valued Neutrosophic Linguistic Reducible Weighted Dual Maclaurin Symmetric Mean.
\end{tabular}

Table 6. The characteristic comparisons of various Single-Valued Neutrosophic Linguistic (SVNL) Aggregation Operator $(\mathrm{AO})$.

\begin{tabular}{|c|c|c|c|}
\hline $\begin{array}{c}\text { Aggregation } \\
\text { operators }\end{array}$ & $\begin{array}{c}\text { Consider } \\
\text { interrelationships } \\
\text { between two } \\
\text { arguments? }\end{array}$ & $\begin{array}{c}\text { Make the } \\
\text { information aggregation } \\
\text { more flexible by } \\
\text { a parameter? }\end{array}$ & $\begin{array}{c}\text { Consider } \\
\text { interrelationships } \\
\text { among multiple } \\
\text { arguments? }\end{array}$ \\
\hline$[13]$ & No & No & No \\
\hline [13] & No & No & No \\
\hline$[17]$ & No & No & No \\
\hline$[16]$ & No & Yes & No \\
\hline$[16]$ & No & Yes & No \\
\hline$[16]$ & No & Yes & No \\
\hline$[15]$ & Yes & No & No \\
\hline$[18]$ & Yes & No & No \\
\hline$[18]$ & Yes & No & No \\
\hline [18] & Yes & No & No \\
\hline SVNLRWMSMa $^{a}$ & Yes & Yes & Yes \\
\hline SVNLRWDMSM $^{\mathrm{b}}$ & Yes & Yes & Yes \\
\hline
\end{tabular}

a SVNLRWMSM: Single-Valued Neutrosophic Linguistic Reducible Weighted Maclaurin Symmetric Mean;

bSVNLRWDMSM: Single-Valued Neutrosophic Linguistic Reducible Weighted Dual Maclaurin Symmetric Mean.

AOs. At the same time, they can take the interrelation of multiple criteria into consideration for dealing with MCDM problems.

For a better comparison with certain MSM operators in various vague environments [20-33], we make an overview of them given in Table 7 .

From Table 7, some existing WMSM operators do not have the idempotency. In addition, the WMSM fails to reduce the MSM when their weights information is equivalent. In other words, it means out of reducibility.

\section{Conclusion}

The dominating contributions are listed as follows:

1. Two novel SVNL AOs including Single-Valued Neutrosophic Linguistic Reducible Weighted MSM (SVNLRWMSM) and Single-Valued Neutrosophic Linguistic Reducible Weighted Dual MSM (SVNLRWDMSM) are presented;

2. Certain properties (monotonicity, idempotency, boundedness, commutativity, and reducibility) are 
Table 7. Characteristic comparisons of various indeterminate environments of Maclaurin Symmetric Mean (MSM).

\begin{tabular}{cccc}
\hline Sets & Aggregation operators & Have the idempotency? & Have the reducibility? \\
\hline SVNLS & SVNLRWMSM & Yes & Yes \\
SVNLS & SVNLRWDMSM & Yes & Yes \\
IFS & {$[20]$} & No & No \\
ULS & {$[21]$} & No & No \\
ILS & {$[22]$} & No & No \\
IULS & {$[22]$} & No & No \\
LIFS & {$[23]$} & No & No \\
LIFS & {$[23]$} & No & No \\
PFS & {$[24]$} & No & No \\
HFLS & {$[25]$} & No & No \\
PFS & {$[26]$} & No & No \\
q-ROFS & {$[26]$} & Yes & No \\
q-ROFS & {$[26]$} & Yes & No \\
q-ROFS & {$[33]$} & Yes & No \\
HFS & {$[27]$} & No & No \\
SVTNS & {$[28]$} & No & No \\
INLS & {$[29]$} & No & No \\
SVNI2TLS & {$[30]$} & No & No \\
2TLS & {$[31]$} & No & No \\
\hline
\end{tabular}

Note: SVNLRWMSM: Single-Valued Neutrosophic Linguistic Reducible Weighted MSM, SVNLRWDMSM: Single-Valued Neutrosophic Linguistic Reducible Weighted Dual MSM, 2TLS: 2-Tuple Linguistic Set, INLS: Interval Neutrosophic Linguistic Set, IFS: Intuitionistic Fuzzy Set, ILS: Intuitionistic Linguistic Set, IULS: Intuitionistic Uncertain Linguistic Set,

LIFS: Linguistic Intuitionistic Fuzzy Set, HFLS: Hesitant Fuzzy Linguistic Set, HFS: Hesitant Fuzzy Set, PFS: Pythagorean Fuzzy Set, q-ROFS: q-rung Orthopair Fuzzy Set, SVN-I2TLS: Single-Valued Neutrosophic Interval 2-Tuple Linguistic Set, SVNLS: Single-Valued Neutrosophic Linguistic Set, SVTNS: Single-Valued Trapezoidal Neutrosophic Set, ULS: Uncertain Linguistic Set.

explored under SVNL environment. Certain existing Maclaurin Symmetric Mean (MSM) operators in various indeterminate environments [20-33] fail to have idempotency or reducibility;

3. Two SVNL Multi-Criteria Decision-Making (MCDM) methods based on SVNLRWMSM and SVNLRWDMSM are introduced. The sensitivity analysis of different $k$ 's on the ranking is discussed (Figure 2 and Table 4);

4. A synthesized appraisal system of six evaluation dimensions is extracted (content experience, interactivity, security, sensitivity, personalization, and travel experience) and it is employed to select the ideal tourism product.

In the future, the SVNLRWMSM and SVNLRWDMSM are applied in computational biology domain such as gene selection $[35,36]$. Besides, we will also put the Reducible Weighted Dual MSM (RWDMSM) and Reducible WMSM (RWMSM) operators into various uncertain circumstances [37-49].

\section{Acknowledgements}

This study was funded by National Natural Science Foundation of China (grant number 61462019), MOE (Ministry of Education in China) Project of Humanities and Social Sciences (grant number 18YJCZH054), and Natural Science Foundation of Guangdong Province (grant number 2018A030307033, 2018A0303130274).

\section{References}

1. China Internet Network Information Center: 42nd China Internet development statistics report. http://www.cac.gov.cn/2018-08/20/c_1123296882. htM (2018).

2. China Tourism Academy: Major data reports on the tourism economy in the first half of 2018. http://www.ctaweb.org/html/2018-8/2018-8-22-1 0-48-50740.html (2018).

3. Wu, Y.F., Hannam, K., and Xu, H.G. "Reconceptu- 
alising home in seasonal Chinese tourism mobilities", Ann. Tour. Res., 73, pp. 71-80 (2018).

4. Lopes, A.P.F., Muñoz, M.M., and Alarcón-Urbistondo, P. "Regional tourism competitiveness using the PROMETHEE approach", Ann. Tour. Res., 73, pp. $1-13$ (2018).

5. Ye, J. "An extended TOPSIS method for multiple attribute group decision making based on single valued neutrosophic linguistic numbers", J. Intell. Fuzzy Syst., 28(1), pp. 247-255 (2015).

6. Smarandache, F., Neutrosophy. Neutrosophic Probability, Set, and Logic:Analytic Synthesis \& Synthetic Analysis, In ProQuest Information \& Learning, F. Smarandache, Edn., 5th Edn., pp. 118-123, American Research Press, Michigan, USA (1998).

7. Wang, H., Smarandache, F., Zhang, Y., et al. "Single valued neutrosophic sets", Multispace Multistruct, 4, pp. 410-413 (2010).

8. Chen, J., Zeng, S., and Zhang, C. "An OWA distancebased, single-valued neutrosophic linguistic topsis approach for green supplier evaluation and selection in low-carbon supply chains", Int. J. Env. Res. Pub. He., 15(7), pp. 1-15 (2018).

9. Ji, P., Zhang, H.Y., and Wang, J.Q. "Selecting an outsourcing provider based on the combined MABACELECTRE method using single-valued neutrosophic linguistic sets", Comput. Ind. Eng., 120, pp. 429-441 (2018).

10. Luo, S., Cheng, P., Wang, J.Q., et al. "Selecting project delivery systems based on simplified neutrosophic linguistic preference relations", Symmetry, $\mathbf{9}(8)$, pp. 1-21 (2017).

11. Peng, X. and Dai, J. "A bibliometric analysis of neutrosophic set: two decades review from 1998 to 2017", Artif. Intell. Rev., 53, pp. 199-255 (2020).

12. Tian, Z., Wang, J., Wang, J., et al. "Simplified neutrosophic linguistic multi-criteria group decisionmaking approach to green product development", Group Decis. Neg., 26(3), pp. 597-627 (2017).

13. Ye, J., Aggregation Operators of Neutrosophic Linguistic Numbers for Multiple Attribute Group Decision Making, SpringerPlus, 5, pp. 1-11 (2016).

14. Tian, Z., Wang, J., Wang, J.Q., et al. "An improved MULTIMOORA approach for multi-criteria decisionmaking based on interdependent inputs of simplified neutrosophic linguistic information", Neural Comput. Appl., 28(1), pp. 585-597 (2017).

15. Tian, Z.P., Wang, J., Zhang, H., et al. "Simplified neutrosophic linguistic normalized weighted Bonferroni mean operator and its application to multicriteria decision-making problems", Filomat, 30(12), pp. 3339-3360 (2016).

16. Tan, R., Zhang, W., and Chen, S. "Some generalized single valued neutrosophic linguistic operators and their application to multiple attribute group decision making", J. Syst. Sci. Inf., 5(2), pp. 148-162 (2017).
17. Wang, J., Tian, C., Zhang, X., et al. "Multi-criteria decision-making method based on simplified neutrosophic linguistic information with cloud model", Symmetry, 10(6), pp. 1-23 (2018).

18. Wang, J.Q., Yang, Y., and Li, L. "Multi-criteria decision-making method based on single-valued neutrosophic linguistic Maclaurin symmetric mean operators", Neural Comput. Appl., 30(5), pp. 1529-1547 (2018).

19. Maclaurin, C. "A second letter to Martin Folkes, Esq.; concerning the roots of equations, with demonstration of other rules of algebra", Philos. Trans. Roy. Soc. London Ser. A., 36, pp. 59-96 (1729).

20. Qin, J. and Liu, X. "An approach to intuitionistic fuzzy multiple attribute decision making based on Maclaurin symmetric mean operators", J. Intell. Fuzzy Syst., 27(5), pp. 2177-2190 (2014).

21. Qin, J. and Liu, X. "Approaches to uncertain linguistic multiple attribute decision making based on dual Maclaurin symmetric mean", J. Intell. Fuzzy Syst., 29(1), pp. 171-186 (2015).

22. Ju, Y., Liu, X., and Ju, D. "Some new intuitionistic linguistic aggregation operators based on Maclaurin symmetric mean and their applications to multiple attribute group decision making", Soft Comput., 20(11), pp. $4521-4548$ (2016).

23. Liu, P. and Qin, X. "Maclaurin symmetric mean operators of linguistic intuitionistic fuzzy numbers and their application to multiple-attribute decisionmaking", J. Exp. Theor. Artif. Intell., 29(6), pp. 11731202 (2017).

24. Yang, W. and Pang, Y. "New Pythagorean fuzzy interaction Maclaurin symmetric mean operators and their application in multiple attribute decision making", IEEE Access, 6, pp. 39241-39260 (2018).

25. Yu, S.M., Zhang, H.Y., and Wang, J.Q. "Hesitant fuzzy linguistic Maclaurin symmetric mean operators and their applications to multi-criteria decisionmaking problem", Int. J. Intell. Syst., 33(5), pp. 953982 (2018).

26. Wei, G. and Lu, M. "Pythagorean fuzzy Maclaurin symmetric mean operators in multiple attribute decision making", Int. J. Intell. Syst., 33(5), pp. 10431070 (2018).

27. Qin, J., Liu, X., and Pedrycz, W. "Hesitant fuzzy Maclaurin symmetric mean operators and its application to multiple-attribute decision making", Int. J. Fuzzy Syst., 17(4), pp. 509-520 (2015).

28. Liu, P. and Zhang, X. "Some Maclaurin symmetric mean operators for single-valued trapezoidal neutrosophic numbers and their applications to group decision making", Int. J. Fuzzy Syst., 20(1), pp. 45-61 (2018).

29. Geng, Y., Wang, X., Li, X., et al. "Some interval neutrosophic linguistic Maclaurin symmetric mean operators and their application in multiple attribute decision making", Symmetry, 10(4), p. 127 (2018). 
30. Ju, D., Ju, Y., and Wang, A. "Multiple attribute group decision making based on Maclaurin symmetric mean operator under single-valued neutrosophic interval 2tuple linguistic environment", J. Intell. Fuzzy Syst., 34(4), pp. 2579-2595 (2018).

31. Zhang, Z., Wei, F., and Zhou, S. "Approaches to comprehensive evaluation with 2-tuple linguistic information", J. Intell. Fuzzy Syst, 28(1), pp. 469-475 (2015).

32. Wei, G.W., Wei, C., Wang, J., et al. "Some q-rung orthopair fuzzy Maclaurin symmetric mean operators and their applications to potential evaluation of emerging technology commercialization", Int. J. Intell. Syst., 34(1), pp. 50-81 (2019).

33. Liu, P., Chen, S.M., and Wang, P. "Multipleattribute group decision-making based on q-rung orthopair fuzzy power Maclaurin symmetric mean operators", IEEE Trans. Syst. Man Cyber. Syst., 50, pp. 3741-3756 (2020). URL https://doi.org/10.1109/ TSMC.2018.2852948

34. Shi, M. and Xiao, Q. "Intuitionistic fuzzy reducible weighted Maclaurin symmetric means and their application in multiple-attribute decision making", Soft Comput., 23(20), pp. 10029-10043 (2019).

35. Huang, H.H. and Liang, Y. "Hybrid L1/2+ 2 method for gene selection in the Cox proportional hazards model", Comput. Meth. Prog. Bio., 164, pp. 65-73 (2018).

36. Huang, H.H. and Liang, Y. "An integrative analysis system of gene expression using self-paced learning and SCAD-Net", Expert Syst. Appl., 135, pp. 102-112 (2019).

37. Peng, X. and Liu, C. "Algorithms for neutrosophic soft decision making based on EDAS, new similarity measure and level soft set", J. Intell. Fuzzy Syst., 32(1), pp. 955-968 (2017).

38. Zhan J. and Alcantud, J.C.R. "A survey of parameter reduction of soft sets and corresponding algorithms", Artif. Intell. Rev., 52(3), pp. 1839-1872 (2019).

39. Peng, X. and Dai, J. "Approaches to single-valued neutrosophic MADM based on MABAC, TOPSIS and new similarity measure with score function", Neural Comput. Appl., 29(10), pp. 939-954 (2018).

40. Peng, X. and Dai, J. "Algorithms for interval neutrosophic multiple attribute decision-making based on MABAC, similarity measure, and EDAS", Int $J$. Uncertain. Quan., 7(5), pp. 395-421 (2017).

41. Liu, P. and Wang, P. "Multiple-attribute decisionmaking based on Archimedean Bonferroni operators of q-rung orthopair fuzzy numbers", IEEE Trans. Fuzzy Syst., 27(5), pp. 834-848 (2019).
42. Liu, P., Zhu, B., Wang, P., et al. "An approach based on linguistic spherical fuzzy sets for public evaluation of shared bicycles in China", Eng. Appl. Artif. Intell., 87, p. 103295 (2020).

43. Liu, P., Chen, S., and Wang, Y. "Multiattribute group decision making based on intuitionistic fuzzy partitioned Maclaurin symmetric mean operators", Inform. Sci., 512, pp. 830-854 (2020).

44. Liu, P. and Liu, W. "Bonferroni harmonic mean operators based on two-dimensional uncertain linguistic information and their applications in land utilization ratio evaluation", Sci. Iran., 26(2), pp. 975-995 (2019).

45. Liu, P. "Maclaurin symmetric means for linguistic Z-numbers and their application to multipleattribute decision making", Sci. Iran., 28(5), pp. 2910-2925 (2020). URL https://doi.org/10.24200/ SCI.2019.5307.1194

46. Peng, X. "New operations for interval-valued Pythagorean fuzzy set", Sci. Iran., 26(2), pp. 1049-1076 (2019).

47. Peng, X. and Selvachandran, G. "Pythagorean fuzzy set: state of the art and future directions", Artif. Intell. Rev., 52(3), pp. 1873-1927 (2019).

48. Wang, L. and Li, N. "Pythagorean fuzzy interaction power Bonferroni mean aggregation operators in multiple attribute decision making", Int. J. Intell. Syst., 35, pp. 150-183 (2020).

49. Lin, M., Wang, H., and Xu, Z. "TODIM-based multi-criteria decision-making method with hesitant fuzzy linguistic term sets", Artif. Intell. Rev., 53(5), pp. 3647-3671 (2020). URL https://doi.org/ $10.1007 / \mathrm{s} 10462-019-09774-9$

\section{Biography}

Xindong Peng works at the School of Information Science and Engineering, Shaoguan University as a lecturer. More than 30 SCI-indexed papers have been published in high-quality international journals including "Applied Soft Computing", "International Journal of Intelligent Systems", and "Artificial Intelligence Review". He is a reviewer of "IEEE Transactions on Fuzzy Systems", "Knowledge Based Systems", "International Journal of Fuzzy Systems", "Technological and Economic Development of Economy", and "Artificial Intelligence Review". His current research interests include neutrosophic set, soft computing, multi-criteria decision-making, pattern recognitions, and soft set theory. 